\title{
Guidelines for lupus anticoagulant testing in South Africa
}

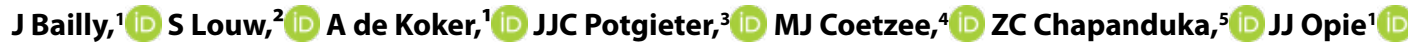 \\ ${ }^{1}$ Division of Haematology, Department of Pathology, Faculty of Health Sciences, University of Cape Town and National \\ Health Laboratory Service, Groote Schuur Hospital, South Africa \\ ${ }^{2}$ Department of Molecular Medicine and Haematology, Faculty of Health Sciences, University of the Witwatersrand and \\ National Health Laboratory Service Charlotte Maxeke Academic Hospital, South Africa \\ ${ }^{3}$ Department of Haematology, Faculty of Health Sciences, University of Pretoria and National Health Laboratory Service, \\ Steve Biko Academic Hospital, South Africa \\ ${ }^{4}$ Department of Haematology and Cell Biology, Faculty of Health Sciences, University of the Free State and National \\ Health Laboratory Service, Universitas Academic Laboratories, South Africa \\ ${ }^{5}$ Division of Haematological Pathology, Faculty of Medicine and Health Sciences, Stellenbosch University and National \\ Health Laboratory Service, Tygerberg Hospital, South Africa
}

Corresponding author, email: jenique.bailly@nhls.ac.za

\begin{abstract}
The lupus anticoagulant (LA) refers to the prolongation of certain coagulation tests due to the action of heterogenous autoantibodies. However, the LA is a misnomer since it is associated with thrombosis in vivo, and most commonly is detected as an incidental, transient laboratory finding associated with conditions such as autoimmune diseases, infections and even in healthy individuals. Repeatedly positive LA testing in the setting of thrombotic and/or obstetric complications is required to diagnose the antiphospholipid syndrome (APS). This review intends to provide clarity on LA testing in the South African context and to provide a national guideline in order to standardise LA testing, interpretation and reporting of results.
\end{abstract}

Keywords: lupus anticoagulant, antiphospholipid syndrome, laboratory testing, mixing studies

\section{Introduction}

Antiphospholipid antibodies (aPLs) are found in a wide range of clinical conditions including autoimmune disorders, infections (viral, bacterial and parasitic), drug exposure, lymphoproliferative disorders and in a proportion of otherwise healthy individuals. ${ }^{1}$ These antibodies belong to a heterogeneous group of autoantibodies usually directed against negatively charged phospholipids bound to plasma proteins, particularly beta-2glycoprotein I ( $\beta 2 \mathrm{GPI})$. The aPLs result in prolongation of the clotting times of phospholipid-dependent coagulation assays in the laboratory, such as the activated partial thromboplastin time (aPTT) and dilute Russell's viper venom time (dRVVT). Despite the prolonged clotting times in vitro, patients with aPLs are predisposed to venous or arterial thromboses, as well as pregnancy loss. ${ }^{2}$ The in vitro coagulation cascade, including the phospholipid dependent components, and laboratory assays used to assess these pathways, is depicted in Figure $1 .{ }^{3}$ Phospholipids play an integral role in progression of coagulation to fibrin clot formation.

Antiphospholipid syndrome (APS) is an acquired autoimmune disorder and represents the most frequently encountered thrombophilic condition in females of childbearing age. ${ }^{4}$ APS is characterised by thromboses and/or adverse obstetric outcomes in a patient with persistent aPLs. These aPLs can be demonstrated by functional lupus anticoagulant (LA) tests and/or by enzymelinked immunosorbent assay (ELISA) methods, which detect antibodies directed against various proteins including cardiolipin

Table l: Revised international consensus diagnostic criteria for definitive anti-phospholipid syndrome ${ }^{8}$

At least one laboratory and one clinical criterion must be met before a diagnosis of antiphospholipid syndrome (APS) can be made. Clinical and laboratory criteria cannot be more than five years apart.

Laboratory criteria* Clinical criteria

1. Positive plasma lupus anticoagulant demonstrated on two occasions at least 12 weeks apart ${ }^{*}$

2. Presence of anticardiolipin antibody of either $\lg \mathrm{M}$ and/ or lgG isotype present at medium or high titres (i.e. $>40 \mathrm{MPL}$ or GPL; or $>99^{\text {th }}$ percentile) on two occasions measured at least 12 weeks apart $^{\dagger}$.

3. Presence of anti- $\beta 2$ glycoprotein I antibody of either IgM and/or lgG with a titre $>99^{\text {th }}$ percentile, on two occasions measured at least 12 weeks apart ${ }^{\dagger}$.
Vascular thrombosis

- One or more objectively confirmed venous, arterial and/or small vessel thrombosis (in the absence of significant vessel wall inflammation ${ }^{\ddagger}$,

Pregnancy complications

- One or more unexplained deaths of a morphologically normal foetus at $\geq 10$ weeks gestation.

One or more premature births of a morphologically normal foetus before 34 weeks gestation and secondary to pre-eclampsia/eclampsia or features of placental insufficiency.

- Three or more spontaneous unexplained pregnancy losses before 10 weeks gestation with no identifiable secondary cause.

* Patients should be classified according to the number of laboratory criteria met. ** Lupus anticoagulant should be detected according to international guidelines, 9,10

t Measured by a standardised enzyme linked immunosorbent assay (ELISA).

‡ Superficial thrombosis is excluded.

MPL - IgM antiphospholipid units; GPL - IgG antiphospholipid units. 


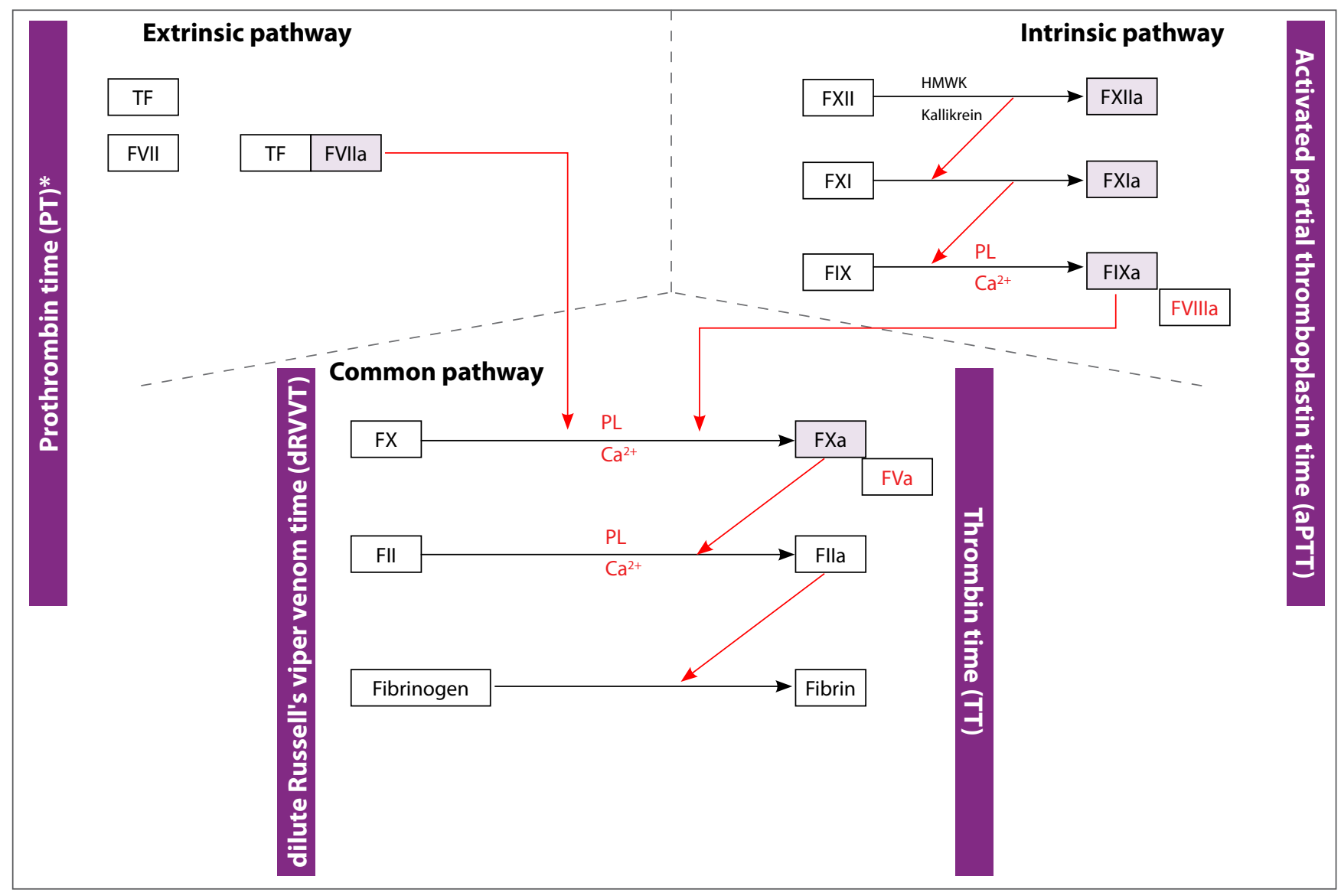

Figure 1: The in vitro coagulation cascade. Adapted from Levine et a ${ }^{3}$

TF - tissue factor; F - coagulation factor; a - activated; HMWK - high-molecular-weight kininogen; PL - phospholipid. * PT is converted to the international normalised ratio (INR)

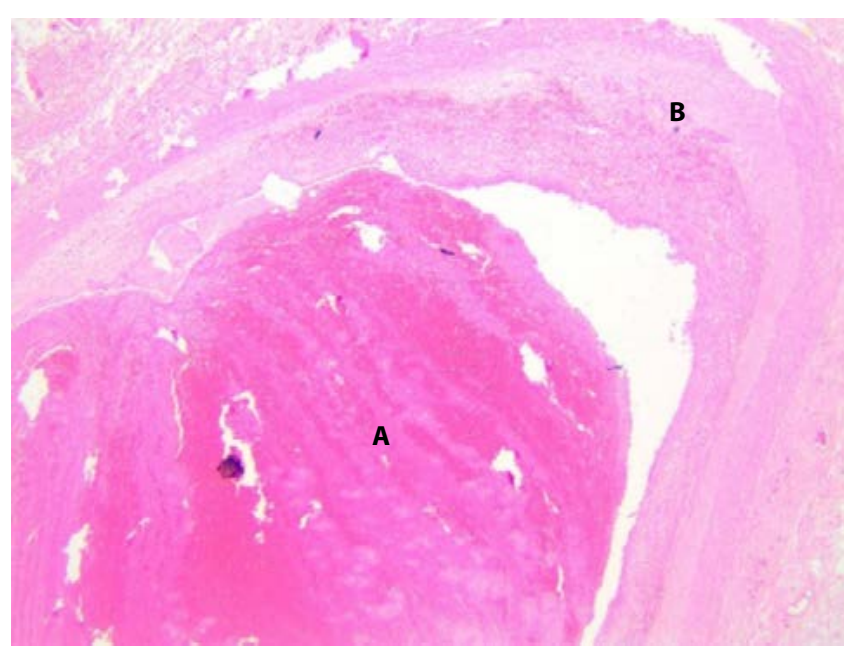

Figure 2: Haematoxylin and eosin (H\&E) image (x 20 magnification) showing adherent thrombus (A) in the deep vein (B) of the left leg, in a patient in whom a pulmonary embolus was found at autopsy

$(\mathrm{aCL})$ and beta-2-glycoprotein I (anti- $\beta 2 \mathrm{GPI})$. Thrombotic events may occur anywhere in the vascular tree, and affect vessels of all sizes (small to large) in both the arterial and/or venous compartments, in any organ system or tissue of the body (Figure 2). Adverse pregnancy outcomes occur secondary to placental insufficiency, which may result in recurrent early pregnancy loss (before 10 weeks gestation), pre-eclampsia/eclampsia with premature birth, or late foetal demise.,.$^{2,5,6}$

The pathogenesis of APS remains poorly understood. Some individuals with persistent aPLs do not develop clinical complications suggesting that additional triggers are required such as an inflammatory insult, or host susceptibility to thrombotic complications. ${ }^{2}$ Vascular endothelial dysfunction and activation of inflammatory responses including the complement system play an important role in the pathogenesis of APS., ${ }^{2,7}$ The international diagnostic criteria for APS stipulate the requirement for at least one clinical and one laboratory criterion to be fulfilled on two occasions at least 12 weeks apart (Table I). ${ }^{8} \mathrm{~A}$ schematic representation of the diagnostic criteria for APS is presented in Figure 3.

Antiphospholipid syndrome may occur in isolation (primary APS) or secondary to an underlying disorder such as systemic lupus erythematosus (SLE), rheumatoid arthritis, haematological malignancy or a solid tumour. ${ }^{2,711}$ Associated features other than thromboses include thrombocytopenia, livedo reticularis,

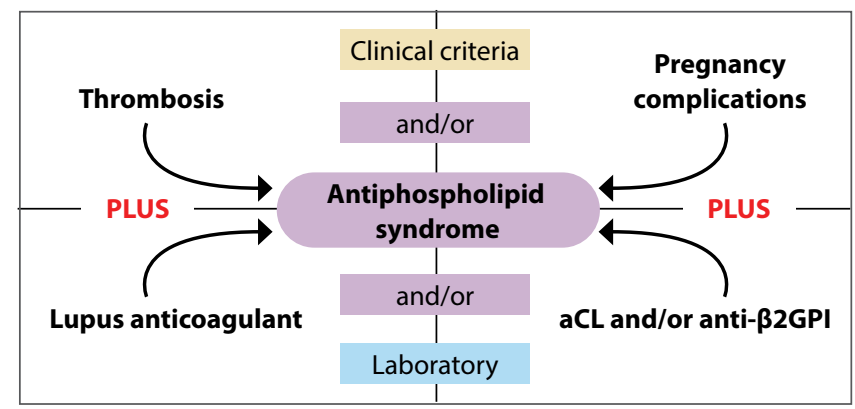

Figure 3: A schematic representation of the international diagnostic criteria for antiphospholipid syndrome ${ }^{8}$

$\mathrm{aCL}$ - anticardiolipin antibodies; anti- $\beta 2 \mathrm{GPI}$ - anti-beta 2-glycoprotein I antibodies. 
valvular heart disease, neurological manifestations and nephropathy. 2,11,12 Catastrophic antiphospholipid syndrome (CAPS) is a rare and life-threatening form of APS, occurring in $<1 \%$ of cases. ${ }^{7}$ Preliminary diagnostic criteria for CAPS include persistent $\mathrm{aPLs}$ in a patient with multi-organ involvement affecting three or more sites simultaneously or within seven days of each other, and with histological evidence of small vessel occlusion with or without vasculitis. ${ }^{13}$

The laboratory diagnosis of these antibodies needs to be made according to established international criteria in accredited laboratories. Patients diagnosed with APS are typically placed on lifelong anticoagulation therapy, which has considerable risks. Testing for antiphospholipid antibodies, including the $\mathrm{LA}, \mathrm{aCL}$ and anti- $\beta 2 \mathrm{GPI}$, is recommended where the clinical diagnostic criteria for APS are met; or in otherwise healthy individuals with an unexpected prolonged activated partial thromboplastin time (aPTT) that does not correct with mixing studies.,12 The anticoagulant effect associated with aPLs represents an in vitro phenomenon caused by the action of these autoantibodies on the proteins of the coagulation cascade which results in prolonged clotting times in the laboratory. According to international criteria, ${ }^{11}$ two different phospholipid dependent clotting assays must be performed when testing for the presence of aPLs and only one of these assays is required to be positive to confirm the diagnosis.

The aim of this review is to provide a national guideline for lupus anticoagulant (LA) testing in the South African context and is intended to standardise testing, interpretation and reporting of results. Key aspects of the pre-analytical, analytical and postanalytical phases of testing are highlighted.

\section{Laboratory testing for a lupus anticoagulant and antiphospholipid antibodies}

Obtaining both functional (clot-based) assays and solid phase immuno-assays, namely enzyme-linked immunosorbent assays (ELISA), is needed for the diagnosis or exclusion of APS. To assess for the presence of aPLs, testing for the $\mathrm{LA}, \mathrm{aCL}$ (IgM and/or IgG) and anti- $\beta 2$ GPI (IgM and/or lgG), is indicated. Positive test results should be documented on two occasions at least 12 weeks apart to rule out transient positive and clinically insignificant results. ${ }^{5}$

\section{Sample preparation}

For coagulation testing, fresh venous blood samples obtained via atraumatic venepuncture must be collected in citrate tubes (0.109M trisodium citrate; 9-parts whole blood to 1 part citrate). Double centrifugation at $4000 \mathrm{rpm}(2000 \mathrm{~g})$ for 15 minutes at room temperature $\left(15\right.$ to $\left.25{ }^{\circ} \mathrm{C}\right)$ should be performed as soon as possible, and ideally within one hour of collection to ensure a platelet count of $<10 \times 10^{9} / \mathrm{L}$ prior to LA testing. ${ }^{6,9,11,14}$ This centrifugation step removes platelets which are a source of phospholipid, as even low concentrations of phospholipid interfere with LA testing. Furthermore, centrifugation is performed at room temperature in order to avoid platelet activation and fragmentation, both of which will increase the phospholipid content of the sample and shorten the clotting times, thus leading to a false negative LA test result. Ultracentrifugation of the sample should be avoided, as it generates phospholipid rich microparticles.13,14 After centrifugation, the plasma is removed without disturbing the white cell and platelet rich buffy layer. The plasma is aliquoted and frozen at between -70 and $-80^{\circ} \mathrm{C}$, unless testing will be performed within four hours of collection. On the day of testing, the frozen aliquoted plasma samples are thawed at $37{ }^{\circ} \mathrm{C}$ in a water bath for approximately five minutes and mixed gently before testing. Testing should not be performed on samples that have undergone more than one freeze-thaw cycle. ${ }^{6,9,12,13}$

\section{Functional LA testing work flow}

A consensus, national LA testing flowchart is presented in Figure 4. This flowchart highlights the key steps in functional laboratory testing required for the identification of an $L A$.

\section{Baseline coagulation tests for the investigation of an $L A$ [or a lupus anticoagulant]}

An international normalised ratio (INR) and activated partial thromboplastin time (aPTT) should be routinely performed. We therefore recommend that at least two citrate tubes appropriately filled to the minimal indicator line are submitted in order to ensure sufficient sample for both the baseline coagulation testing and LA testing. These baseline coagulation tests exclude patient exposure to anticoagulants or factor deficiencies that may influence interpretation of LA test results. Testing for LA is advised prior to starting anticoagulant therapy, or at least three weeks after their discontinuation, since anticoagulant therapy may interfere with interpretation of LA testing results. If the aPTT is prolonged, a 50:50 mixing study with normal plasma is necessary. If an LA is present in the sample, the aPTT will typically not correct with mixing, but if a factor deficiency is the cause for the aPTT prolongation, it will. When the aPTT is prolonged and does not correct with mixing, a thrombin time (TT) is advised to exclude the presence of unfractionated, and to a lesser extent low molecular weight heparin or direct thrombin inhibitors. ${ }^{12}$ Many dilute Russell's viper venom time (dRVVT) reagents do however include a heparin neutralising agent that should be sufficient to neutralise a prophylactic dose of low molecular weight heparin (LMWH). ${ }^{9,11}$

The dRVVT assay utilises reagents which directly activate Factor $X(F X)$ in the common coagulation pathway, an action similar to that of Russell's viper snake venom (see Figure 1). The direct activation of FX leads to fibrin clot formation, and deficiency in the other clotting factors that usually activate FX will therefore not affect dRVVT results. ${ }^{10}$ The International Society on Thrombosis and Haemostasis (ISTH) recommends that LA testing should be performed only if the INR is $<1.5$. It recommends that where clinically appropriate LA testing should be postponed until warfarin therapy has been safely stopped and the INR has normalised. Alternatively, LA testing can be performed after three months of adequate warfarin therapy by switching the patient to appropriate doses of LMWH. Warfarin should only be stopped to allow LA testing, if testing is essential on clinical or therapeutic grounds. ${ }^{9,11}$ The direct oral anticoagulants (DOACs), including dabigatran, rivaroxaban, edoxaban and apixaban, also 


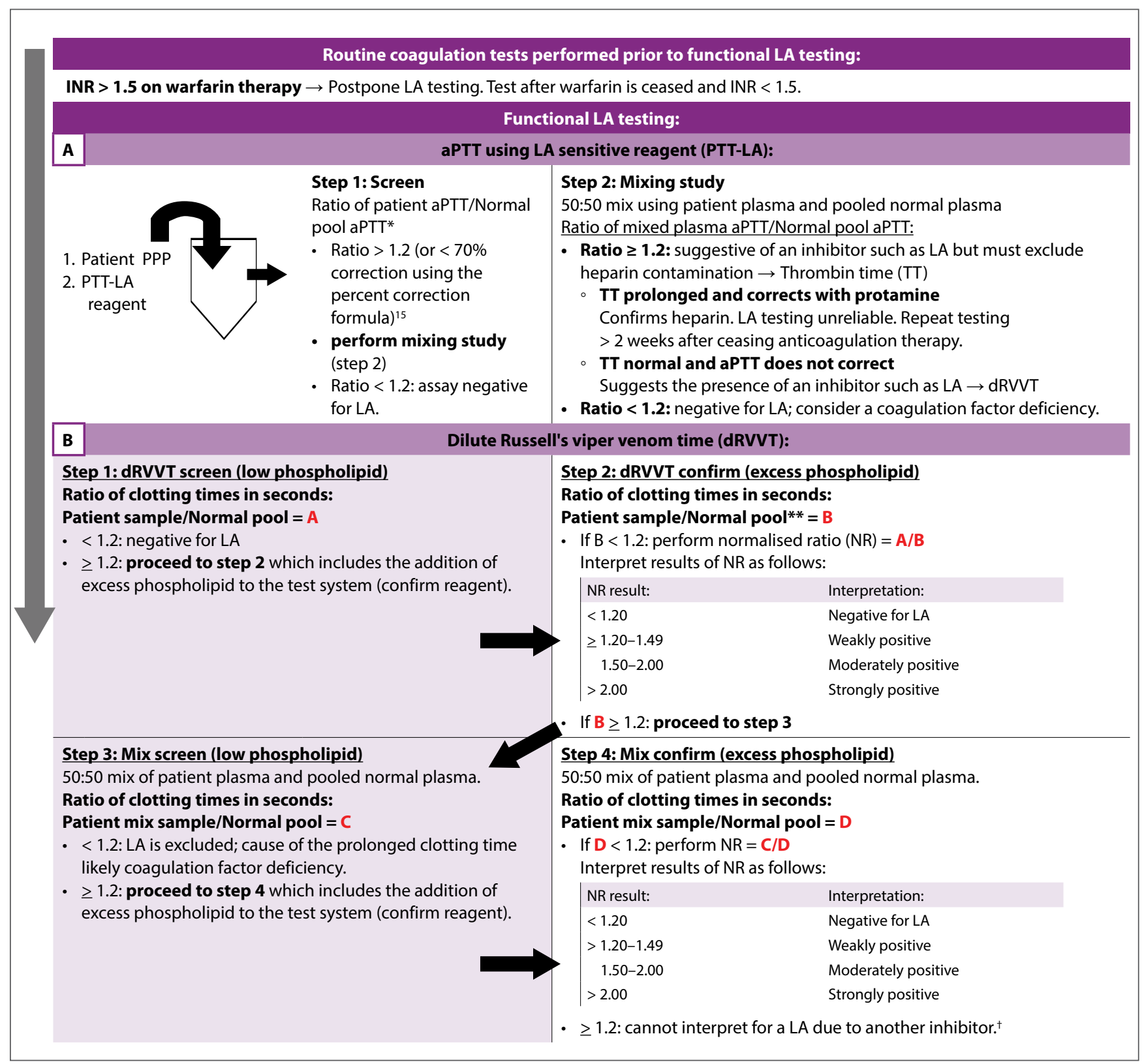

Figure 4: Proposed national LA testing flowchart

LA - lupus anticoagulant; INR - international normalised ratio; aPTT - activated partial thromboplastin time; TT - thrombin time; PPP - platelet poor plasma; $\mathrm{PL}$ - phospholipid; aPLs - antiphospholipid antibodies.

*Normal pool: control normal plasma. ${ }^{* *}$ The effect of excess phospholipid used in the confirm step of the dRVVT can also be measured using the equation [(screen clotting time - confirm clotting time)/screen clotting time) x 100], where a \% correction above the local cut-off value confirms the presence of LA. ${ }^{9+}$ Other inhibitors such as FVIII specific inhibitor or heparin may cause these results.

interfere with LA testing. The dRVVT is particularly sensitive to their interference and LA testing is therefore not recommended while the patient is on DOAC therapy. ${ }^{16,17}$

\section{Functional assays to detect a lupus anticoagulant}

International criteria require that two phospholipid-dependent coagulation assays, based on different test principles, are used to diagnose an LA.9,10,12,13 Most guidelines recommend using the dRVVT, as well as an aPTT done with a lupus sensitive reagent (PTT-LA), as explained below. If either test result is prolonged and fails to correct by mixing with either normal pooled plasma (NPP) or commercial normal plasma, while correcting with the addition of excess phospholipid, the LA test is considered positive.9,10 In summary, three steps are indicated to investigate for the presence of LA:9,13
1. a screening step with low phospholipid in the test system;

2. a confirmatory step with excess of phospholipid in the test system; and

3. a mixing step.

The PTT-LA test may only include a screening and mixing step i.e. step 1 and step 3 above, without a confirmatory phospholipidrich step i.e. step 2, if no reagent with excess phospholipid is available to the testing laboratory. Most laboratories in South Africa do not have a PTT-confirm (phospholipid rich) reagent readily available.

\section{A. LA sensitive aPTT (PTT-LA]}

The 'LA sensitive' assay refers to the low concentration of phospholipid in the test system, which is relatively insensitive to 
low concentrations of heparin or factor deficiency. ${ }^{9,14}$ The result is reported as a ratio between the patient's aPTT (PTT-LA) and an aPTT obtained with the NPP (or commercial normal plasma). Most laboratories perform only a screening test, followed by a mixing study if the screen/normal pool aPTT ratio is $\geq 1.2$ (Figure 4). A positive result $(\geq 1.2)$ after aPTT mixing studies suggests the presence of an inhibitor such as an LA, high dose heparin therapy, sample contamination or more rarely a specific inhibitor to a coagulation factor in the intrinsic pathway (most commonly a Factor VIII inhibitor). ${ }^{9}$

A number of formulae including the percent correction formula ${ }^{18,19}$ and the Rosner index, ${ }^{20}$ may be used to assess the difference in the clotting times obtained before and after performing mixing studies. Certain cut-off values are suggestive of an inhibitor, ${ }^{15}$ however none of these formulae are $100 \%$ sensitive or specific. When using the percent correction formula, a cut-off value of $<70 \%$ suggests the presence of an inhibitor; whereas $>70 \%$ correction suggests a factor deficiency. ${ }^{18,19}$ In one large study, the percentage correction was shown to be slightly more sensitive to detecting the LA than the Rosner index (95\% versus $93 \%) .{ }^{15}$

$$
\text { Percent correction }=\frac{\text { test aPTT }-50: 50 \text { mix aPTT }}{\text { test aPTT }- \text { normal aPTT }} \times 100
$$

\section{Rosner index $=\frac{50: 50 \text { mix aPTT }- \text { normal aPTT }}{\text { test aPTT }} \times 100$}

\section{B. Dilute Russell's viper venom time [dRVVT]}

The dRVVT (Figure 1) assesses the effect of LA on the common coagulation pathway via the direct activation of FX in a calciumdependent manner. Activation of FX by the procoagulant snake venom-based reagent is independent of the concentration of phospholipid in the test system. Thus testing under phospholipid poor (screen step) and phospholipid rich (confirm step) conditions is feasible. ${ }^{10}$ If the screen step ratio result is increased $(\geq 1.2)$, the confirm step excludes the presence of an LA by assessing phospholipid dependence, and further defines the strength of
LA activity. ${ }^{9,12}$ Phospholipid dependence is demonstrated by a significant shortening in the clotting time in the confirm step as compared to the screen step, and a normalised LA ratio reported to two decimal places should be calculated:9,10

Normalised LA ratio $=\frac{\text { Screen ratio } A}{\text { Screen ratio } B}$

Depending on the results of the screen and confirm steps, a mixing step may be needed to further assess for other causes of the prolonged clotting times, such as clotting factor deficiencies or the presence of another inhibitor such as heparin. The mixing step is indicated if the confirm step ratio is $\geq 1.2$. Mixing studies are performed by adding either NPP or commercial normal plasma in a 1:1 ratio to the patient's plasma, which is then done without a pre-incubation step, within 30 minutes of preparing the mix. The NPP (or commercial normal plasma) supplements any deficient clotting factor to at least $50 \%$ of normal. Therefore, NPP (or commercial normal plasma) should normalise the clotting time of the mixing study in the absence of an inhibitor, and if the original prolongation in the clotting time was due to coagulation factor deficiency. If the clotting time remains prolonged at a ratio of $\geq 1.2$ compared to that of NPP (or commercial normal plasma), the mixed plasma must be analysed with a phospholipid rich confirm reagent to detect the presence of an LA in combination with a factor deficiency. It is important to note that a very weak LA may correct with the mixing study prior to the confirmation step, resulting in a false negative LA result.9,12

\section{Reporting of results}

Patient results for the screen, confirm and mixing steps are reported as a ratio between the clotting times obtained for the patient's plasma and that of the NPP (or commercial normal plasma) (Figure 3).9-11 Interpretive reporting and recommendations for repeat testing should be provided as indicated..$^{9110}$ The reports should clearly indicate whether an LA was detected or not, which method was used, and which cut-off values were used. ${ }^{10,11}$ Mixing studies should only be performed if indicated. In the rare instance where a ratio of $\geq 1.2$ is reported

Table II: Dilute Russell's viper venom time (dRVVT) results and their interpretations ${ }^{9}$

\begin{tabular}{|c|c|c|c|c|c|c|}
\hline \multicolumn{3}{|c|}{ Patient plasma } & \multicolumn{3}{|c|}{ Patient/normal pooled plasma mix (1:1) } & \multirow{2}{*}{ Interpretation } \\
\hline Screen & Confirm & Screen NR & Screen mix & Confirm mix & Mix NR & \\
\hline$<1.2$ & - & - & - & - & - & LA not detected \\
\hline$\geq 1.2$ & $<1.2$ & $<1.2$ & - & - & - & LA not detected \\
\hline$\geq 1.2$ & $<1.2$ & $\geq 1.2$ & - & - & - & $\begin{array}{l}\text { LA detected (state whether weak, moderate or strong as } \\
\text { per NR). Repeat after } 12 \text { weeks once off anticoagulation } \\
\text { to demonstrate persistence / significance }\end{array}$ \\
\hline$\geq 1.2$ & $\geq 1.2$ & - & $<1.2$ & - & - & LA not detected. Exclude clotting factor deficiency. \\
\hline$\geq 1.2$ & $\geq 1.2$ & - & $\geq 1.2$ & $<1.2$ & $<1.2$ & LA not detected \\
\hline$\geq 1.2$ & $\geq 1.2$ & - & $\geq 1.2$ & $<1.2$ & $\geq 1.2$ & $\begin{array}{l}\text { LA detected (state whether weak, moderate or strong as } \\
\text { per NR). Repeat after } 12 \text { weeks once off anticoagulation } \\
\text { to demonstrate persistence / significance }\end{array}$ \\
\hline$\geq 1.2$ & $\geq 1.2$ & - & $\geq 1.2$ & $\geq 1.2$ & - & $\begin{array}{l}\text { Unsuitable for interpretation of a LA due to the presence } \\
\text { of another inhibitor such as heparin or coagulation } \\
\text { factor specific inhibitor. Advise repeat testing after } \\
\text { anticoagulation therapy has been ceased. }\end{array}$ \\
\hline
\end{tabular}


for the dRVVT confirm step (step 2), an automatically calculated normalised LA ratio result should be suppressed if it is displayed by the laboratory information system (LIS). If the mixing step ratio is $<1.2$, a weak LA may be present and the dRVVT should be reported as suggestive of a weak LA, with follow-up testing advised once the patient no longer requires anticoagulation. ${ }^{14}$ Various dRVVT results and their appropriate interpretations are provided in Table II.

\section{Quality assurance and establishing cut-off values}

Internal controls, manufacturer's normal (LA negative) and abnormal (LA positive) controls, or patient samples with known LA positive or negative results, should be analysed with each batch of patient tests to ensure the reporting of accurate results. These controls should be handled like patient samples. Results of the normal control should fall within the laboratory-derived cutoff values for a negative LA. ${ }^{11}$ Ideally, local population-specific cut-off values for a positive result should be determined by each laboratory because of local differences in reagents and analysers used. This is not always feasible because of costs, labour and availability of reference controls. It is therefore acceptable to use manufacturer-established cut-off values, or established cut-off values from another laboratory which uses the same reagents, analyser and analytical protocol. A cut-off value should be established for each LA test. Commonly a ratio of the clotting time of the sample vs that of NPP (or commercial normal plasma) of $\geq 1.2$ for both the aPTT (PTT-LA) and dRVVT is taken as indicative of an inhibitor, such as an LA.,13

Table III highlights some key recommendations for avoiding false positive and negative LA test results. ${ }^{6}$ Testing during the acute phase of illness should be avoided. Fibrinogen and Factor VIII are both acute phase reactants and elevated levels of either one (or both) as seen during infection, inflammation and pregnancy, will shorten the clotting times of clot-based assays and can interfere with LA testing. In sepsis or inflammation there is an increase in circulating phospholipid rich platelet microparticles which may also interfere with the detection of LA. Thus testing in the acute phase of illness should be avoided as it may result in a false negative result. 6,10

\section{Solid phase immuno-assays}

An ELISA is typically used to detect anti- $\beta 2$ GPI and $a C L$ antibodies in serum or platelet poor plasma (PPP). The isotype and titres should be determined and reported. ${ }^{21}$ One or both antibodies may be detected with or without a positive LA result and only one of these tests needs to be positive to satisfy diagnostic criteria for APS. Results reported by different laboratories vary enormously and likely reflect the differences in pre-, post- and analytical conditions, the reference materials used for calibration, and imprecision of the method in use. ${ }^{12,21}$ The reader is referred to the latest guidelines from the Scientific and Standardisation Committee (SSC) of the International Society on Thrombosis and Haemostasis (ISTH) on testing for antiphospholipid antibodies with solid phase assays. ${ }^{21}$
Table III: Recommendations for avoiding false positive and negative LA test results ${ }^{6}$

\section{To avoid false positive results:}

1. Both a LA sensitive aPTT, and dRVVT should be performed.

2. Screen-, confirm- and mix steps should be performed as indicated, and local cut-off values should be determined by each laboratory.

3. Fresh NPP prepared from at least 20 normal donor plasmas with an equal male and female distribution should ideally be used with each new batch of samples tested.

4. ELISA assay for both $\mathrm{aCL}$ and anti- $\beta 2 \mathrm{GPI}$ should be performed together with LA testing.

5. A positive LA result should be documented on two occasions at least 12 weeks apart to confirm significance.

6. LA testing should be performed prior or after stopping anticoagulation therapy. Samples should be rejected if the INR is $>1.5$, unless dilutions are performed.

7. Adequate IQC testing is needed for each test batch as well as participation in an EQA programme.

\section{To avoid false negative results:}

1. Avoid testing during the acute phase of illness.

2. Ensure correct plasma handling which includes the appropriate collection of blood, double centrifugation to obtain PPP, quick freezing if samples are not analysed within 4 hours, appropriate thawing technique and gentle mixing of samples prior to analysis.

3. Perform the dRVVT confirmatory step prior to mixing studies to detect a weak LA.

LA - lupus anticoagulant; aPTT - activated partial thromboplastin time; dRVVT - dilute Russell's viper venom time; NPP - normal pooled plasma; PPP - platelet poor plasma; $\mathrm{aCL}$ - anticardiolipin antibodies; anti- $\beta 2 \mathrm{GPI}$ - anti-beta 2 glycoprotein I; INR - international normalised ratio; IQC - internal quality control; EQA - external quality assessment.

\section{Summary}

This guideline aims to standardise testing, interpretation and reporting of functional LA testing in South Africa. Testing for an LA can be challenging as the aPLs are heterogeneous and anticoagulant therapy can interfere with testing. Functional coagulation tests for an LA, and ELISA assays for anti- $\beta 2$ GPI and $\mathrm{aCL}$ antibodies must be performed as part of the laboratory investigation for aPLs. An aPTT obtained with LA sensitive reagents (PTT-LA) and dRVVT are both required to detect an LA since these are two phospholipid-dependent coagulation tests based on different principles. Because of the significant clinical implications of the LA results, testing should only be performed by trained, competent staff in accredited laboratories with appropriate internal controls and EQA programmes.

Baseline coagulation and functional LA tests should be performed prior to starting anticoagulation, or after a sufficient period off anticoagulant therapy. Other important preanalytic factors to consider include the collection of blood samples (atraumatic venepuncture, adequate sample volume and collection in a $0.109 \mathrm{M}$ trisodium citrate tube), double centrifugation of samples at room temperature to obtain PPP with a platelet count of $<10 \times 10^{9} / \mathrm{L}$, quick freezing if samples are not analysed within four hours, correct thawing technique of frozen aliquots and gentle mixing of samples prior to analysis. Figure 4 provides a consensus national LA testing flowchart. This flowchart highlights the key steps in functional laboratory testing for an LA. The interpretation and reporting of results as documented in this guideline, as well the caveats listed in Table II, should be kept in mind. 


\section{ORCID}

J Bailly (D) https://orcid.org/0000-0002-6542-6956

S Louw (iD) https://orcid.org/0000-0002-4315-1496

A de Koker (D) https://orcid.org/0000-0003-0201-007X

JJC Potgieter (D) https://orcid.org/0000-0001-5366-6744

MJ Coetzee (iD https://orcid.org/0000-0003-2762-0966

ZC Chapanduka (iD https://orcid.org/0000-0002-5489-8392

JJ Opie (iD https://orcid.org/0000-0002-1408-2604

\section{References}

1. Love PE, Santoro SA. Antiphospholipid antibodies: anticardiolipin and the lupus anticoagulant in systemic lupus erythematosus (SLE) and in non-SLE disorders. Prevalence and clinical significance. Ann Intern Med. 1990;112(9):682-98. https:// doi.org/10.7326/0003-4819-112-9-682.

2. Arachchillage DRJ, Laffan M. Pathogenesis and management of antiphospholipid syndrome. Br J Haematol. 2017;178(2):181-95. https://doi. org/10.1111/bjh.14632.

3. Levine JS, Branch DW, Rauch J. The antiphospholipid syndrome. N Engl J Med. 2002;346(10):752-63. https://doi.org/10.1056/NEJMra002974.

4. Limper M, Scire CA, Talarico R, et al. Antiphospholipid syndrome: state of the art on clinical practice guidelines. RMD Open. 2018;4(Suppl 1):e000785. https://doi. org/10.1136/rmdopen-2018-000785.

5. Chaturvedi S, McCrae KR. The antiphospholipid syndrome: still an enigma. Hematology Am Soc Hematol Educ Program. 2015;2015:53-60. https://doi. org/10.1182/asheducation-2015.1.53.

6. Devreese KM. Antiphospholipid antibody testing and standardization. Int J Lab Hematol. 2014;36(3):352-63. https://doi.org/10.1111/ijlh.12234.

7. Corban MT, Duarte-Garcia A, McBane RD, et al. Antiphospholipid Syndrome: Role of Vascular Endothelial Cells and Implications for Risk Stratification and Targeted Therapeutics. J Am Coll Cardiol. 2017;69(18):2317-30. https://doi.org/10.1016/j. jacc.2017.02.058.

8. Miyakis $S$, Lockshin MD, Atsumi $T$, et al. International consensus statement on an update of the classification criteria for definite antiphospholipid syndrome (APS). J Thromb Haemost. 2006;4(2):295-306. https://doi. org/10.1111/j.1538-7836.2006.01753.x.

9. Pengo V, Tripodi A, Reber $G$, et al. Update of the guidelines for lupus anticoagulant detection. Subcommittee on Lupus Anticoagulant/
Antiphospholipid Antibody of the Scientific and Standardisation Committee of the International Society on Thrombosis and Haemostasis. J Thromb Haemost. 2009;7(10):1737-40. https://doi.org/10.1111/j.1538-7836.2009.03555.x.

10. CLSI. Laboratory Testing for the Lupus Anticoagulant, Approved Guidelines. CLSI document H60-A Wayne, PA: Clinical and laboratory standards Institute; 2014.

11. Keeling D, Mackie I, Moore GW, Greer IA, Greaves M, British Committee for Standards in Haematology. Guidelines on the investigation and management of antiphospholipid syndrome. Br J Haematol. 2012;157(1):47-58. https://doi. org/10.1111/j.1365-2141.2012.09037.x.

12. Pengo V, Banzato A, Bison E, et al. Laboratory testing for antiphospholipid syndrome. Int J Lab Hematol. 2016;38(Suppl 1):27-31. https://doi.org/10.1111/ ijlh.12507.

13. Lim W. Antiphospholipid syndrome. Hematology Am Soc Hematol Educ Program. 2013;2013:675-80. https://doi.org/10.1182/asheducation-2013.1.675.

14. Moore GW. Recent guidelines and recommendations for laboratory detection of lupus anticoagulants. Semin Thromb Hemost. 2014;40(2):163-71. https://doi. org/10.1055/s-0033-1364185.

15. Devreese KM. Interpretation of normal plasma mixing studies in the laboratory diagnosis of lupus anticoagulants. Thromb Res. 2007;119(3):369-76. https://doi. org/10.1016/j.thromres.2006.03.012.

16. Favaloro EJ, Mohammed S, Curnow J, Pasalic L. Laboratory testing for lupus anticoagulant (LA) in patients taking direct oral anticoagulants (DOACs): potential for false positives and false negatives. Pathology. 2019;51(3):292-300. https://doi.org/10.1016/j.pathol.2018.11.008.

17. Hoxha A, Banzato A, Ruffatti A, Pengo V. Detection of lupus anticoagulant in the era of direct oral anticoagulants. Autoimmun Rev. 2017;16(2):173-8. https://doi. org/10.1016/j.autrev.2016.12.010.

18. Chang SH, Tillema V, Scherr D. A "percent correction" formula for evaluation of mixing studies. Am J Clin Pathol. 2002;117(1):62-73. https://doi.org/10.1309/ RREK-8L6M-D2KC-HWLH.

19. Chang S. More on: normal plasma mixing studies in the laboratory diagnosis of lupus anticoagulant. J Thromb Haemost. 2004;2(8):1480-1. https://doi. org/10.1111/j.1538-7836.2004.00847.x.

20. Rosner E, Pauzner R, Lusky A, Modan M, Many A. Detection and quantitative evaluation of lupus circulating anticoagulant activity. Thromb Haemost. 1987;57(2):144-7. https://doi.org/10.1055/s-0038-1651083.

21. Devreese KM, Pierangeli SS, De Laat B, et al. Testing for antiphospholipid antibodies with solid phase assays: guidance from the SSC of the ISTH. JThromb Haemost. 2014;12(5):792-5. https://doi.org/10.1111/jth.12537. 